\title{
Depressive Rumination and Co-Morbidity: Evidence for Brooding as a Transdiagnostic Process
}

\author{
Edward R. Watkins
}

Published online: 7 August 2009

(C) The Author(s) 2009. This article is published with open access at Springerlink.com

\begin{abstract}
A sample of 116 patients with unipolar mood disorders referred to a specialist research clinic were assessed to investigate (a) whether rumination is a transdiagnostic process that is related to co-morbid Axis I and II symptoms and diagnosis in depressed patients; (b) whether common findings in the depressive rumination literature could be replicated in a recurrent depressed sample. Consistent with the transdiagnostic hypothesis, rumination was positively associated with both depression and anxiety, brooding was related to co-morbid obsessive-compulsive disorder and generalized anxiety disorder, and rumination was associated with traits associated with borderline personality disorder, most notably self-report of unstable relationships and inconsistent sense of self. As predicted, rumination was equivalent in currently depressed and formerly depressed patients, suggesting that rumination is not simply dependent on mood state or clinical status. As predicted, the brooding subtype most strongly correlated with depressed and anxious symptoms, consistent with previous observations that brooding is the more maladaptive form of rumination. As predicted, rumination was associated with reports of sexual abuse. Inconsistent with previous findings, there was no gender difference in rumination.
\end{abstract}

Keywords Depression - Rumination - Personality traits · Brooding ·

Co-morbidity $\cdot$ Borderline personality $\cdot$ Transdiagnostic

\section{Introduction}

Depressive rumination, defined as "behavior and thoughts that focus one's attention on one's depressive symptoms and on the implications of these

\footnotetext{
E. R. Watkins ( $₫)$

Mood Disorders Centre, School of Psychology, Washington Singer Laboratories, University of Exeter, Perry Road, Exeter EX4 4QG, UK e-mail: E.R.Watkins@exeter.ac.uk
} 
symptoms" (Nolen-Hoeksema 1991, p. 569) has been identified as a core process in the onset and maintenance of depression. Prospective longitudinal studies have found that depressive rumination predicts the likelihood, severity and duration of syndromal depression (Nolen-Hoeksema 2000; Spasojevic and Alloy 2001). Experimental studies have found that induction of depressive rumination intensifies dysphoric mood, increases negative thinking and impairs problem-solving relative to distraction in individuals already in a dysphoric mood, but has no differential effect in individuals who are not already dysphoric (e.g., Lyubomirsky and NolenHoeksema 1995; Nolen-Hoeksema and Morrow 1993).

There is also increasing evidence that depressive rumination may be implicated in symptoms and disorders other than depression. Prospective longitudinal studies have found that rumination predicts level of anxiety symptoms as well as depressive symptoms (Hong 2007; Nolen-Hoeksema 2000; Sarin et al. 2005; Segerstrom et al. 2000) and that rumination predicts bulimia and substance abuse in female adolescents (Nolen-Hoeksema et al. 2007). Furthermore, there is evidence that repetitive thought about personal concerns occurs in a number of anxiety disorders including social anxiety (e.g., Abbott and Rapee 2004; Mellings and Alden 2000), generalized anxiety disorder (GAD; American Psychiatric Association 1994; Hoyer et al. 2002), obsessive-compulsive disorder (OCD; Vanoppen et al. 1995) and posttraumatic stress disorder (e.g., Ehlers et al. 1998; Mayou et al. 2002). These findings prompted the hypothesis that rumination is a transdiagnostic process, that is, a cognitive process that causally contributes to the development of a number of different psychiatric disorders, which may explain the high levels of co-morbidity between depression and anxiety (Harvey et al. 2004). However, the relationship between rumination and the diagnosis of disorders co-morbid to depression has not been investigated in currently depressed and formerly depressed patients. Therefore, the first aim of this study was to test the transdiagnostic hypothesis which predicts that rumination will be related to co-morbid symptoms and co-morbid diagnoses in depressed patients.

Moreover, there is also initial evidence of a relationship between rumination and personality dysfunction, also consistent with a transdiagnostic perspective. Participants with depression and co-morbid borderline personality disorder exhibited higher levels of rumination than patients with depression alone or control participants (Abela et al. 2003). In a large sample of undergraduates with high and low cognitive risk for depression but no current Axis I disorders, rumination was not related to personality disorder diagnosis but was uniquely related to scores on borderline and obsessive-compulsive dimensions (Smith et al. 2006). However, these findings have yet to be replicated and have not yet been examined in depressed patients across the full range of personality disorders. Thus, a second and related aim of this study was to further explore the relationship between rumination and dysfunctional interpersonal, behavioral traits associated with personality disorders, and to test whether the prior findings linking rumination to borderline and obsessive-compulsive dimensions (Abela et al. 2003; Smith et al. 2006) could be replicated in a sample of currently depressed and formerly depressed patients. Importantly, this study is only the second study to examine the relationship between rumination and the full range of Axis II dysfunction and the first to examine the 
relationship between rumination and personality dysfunction in a clinical sample that included formerly depressed patients.

This study of rumination within a clinical sample also provided an opportunity to test whether a number of important previous findings in predominantly non-clinical samples could be replicated and extended into a sample of patients with chronic and recurrent depression. First, there is some evidence that depressive rumination has personality trait-like properties rather than being substantively determined by current mood state or clinical status. Thus, in three samples of college students, Roberts and colleagues (1998) found that currently dysphoric and previously dysphoric participants reported elevated levels of depressive rumination relative to neverdysphoric controls, and in two out of the three samples found that currently dysphoric participants did not significantly differ from (prolonged) previously dysphoric participants in their level of rumination. This pattern of results suggests that "rumination might act as a trait characteristic that persists subsequent to remission of dysphoric episodes" (Roberts et al. 1998, p. 407). However, in contrast, a prospective longitudinal study of 88 students who met criteria for recent-onset major depression found that rumination decreased over time in those students who recovered over 6 months, but did not change in those students who did not recover (Kasch et al. 2001). This result suggests that state factors and clinical status do contribute to the level of self-reported rumination. These mixed findings suggest the value of further investigation of whether rumination differs with clinical status (currently vs. formerly depressed), in particular, within a non-student patient sample.

Second, a reliable finding within the rumination literature is that levels of depressive rumination are higher in females than males (Butler and NolenHoeksema 1994; Nolen-Hoeksema and Jackson 2001; Nolen-Hoeksema et al. 1999). This gender difference in rumination might, in part, account for the increased rates of depression in women relative to men (Butler and Nolen-Hoeksema 1994; Nolen-Hoeksema et al. 1999).

Third, recent research has suggested that there are distinct subtypes of depressive rumination that have distinct functional effects. Experimental studies have found that a more abstract and evaluative form of rumination has negative consequences relative to a more concrete and experiential form of rumination on negative global self-evaluations (Rimes and Watkins 2005), social problem solving (Watkins and Moulds 2005), and specificity of autobiographical memory recall (Watkins and Teasdale 2001, 2004). Moreover, factor analysis of the main self-report measure of rumination, the Response Styles Questionnaire (RSQ; Nolen-Hoeksema and Morrow 1991) within a large community sample, revealed two distinct factors, Brooding and Reflection, once items referring to symptoms of depression were removed (Treynor et al. 2003). Brooding measured at baseline predicted both increased concurrent depression and increased future depression assessed 1 year later, even after controlling for depression levels at baseline, whereas reflection measured at assessment predicted increased concurrent depression but reduced prospective depression assessed 1 year later. Thus, brooding appears to have more maladaptive effects than reflection.

Fourth, a retrospective study found that elevated rumination is associated with reports of sexual abuse on a questionnaire asking undergraduate participants about 
the nature and frequency of unwanted and forced sexual activity (Conway et al. 2004). Increased rumination was significantly correlated with the report of greater frequency of unwanted sexual invitations and touching in childhood and with the report of greater frequency of forced sexual acts including rape in adulthood. Although constrained by the typical limitation of retrospective studies (the possibility of mood, memory and/or demand biases influencing report of events), this finding suggests the possibility that sexual abuse might increase risk for rumination.

A limitation of many of these studies reported above is that non-clinical samples, typically undergraduates (e.g., Conway et al. 2004; Kasch et al. 2001; Sarin et al. 2005; Smith et al. 2006) or community samples (Nolen-Hoeksema 2000; Treynor et al. 2003) were used and that depression was not always defined by full diagnostic criteria (e.g., Conway et al. 2004; Roberts et al. 1998). These limitations leave open the question of the generalizability of these findings to a clinical population consisting of depressed patients. Before one can be confident that these reported observations are generalizable and clinically meaningful phenomena, they need to be replicated in patients with clinical depression. Thus, the third aim of the current study was to investigate whether the above findings can be replicated in a consecutive series of depressed patients referred to a research treatment center. Further, many of the studies reported above did not specifically investigate whether the distinct subtypes of rumination had distinct relationships with psychopathology. Thus, a further aim of the current study was to investigate the relationship of brooding and reflection to psychopathology, co-morbidity and report of sexual abuse.

In summary, we used data from consecutive referrals to a mood disorders research treatment clinic to test the following predictions: (a) Consistent with the transdiagnostic hypothesis of rumination, rumination was predicted to be correlated with depression, anxiety and to be associated with the prevalence of disorders comorbid with depression such as OCD, GAD, social anxiety; (b) Also related to the transdiagnostic hypothesis, rumination was predicted to be associated with borderline and obsessive-compulsive personality dimensions; (c) Consistent with the hypothesis that rumination is a personality trait conferring vulnerability, levels of rumination in currently depressed patients were predicted to be equivalent to levels of rumination in formerly depressed patients; (d) Consistent with previous findings, rumination was predicted to be higher in women than men; (e) Consistent with the hypothesis that there are distinct sub-types of rumination with distinct functional effects, the correlations between brooding and symptoms were predicted to be greater than the correlations between reflection and symptoms; (f) Rumination was predicted to be related to reports of sexual abuse.

\section{Methods}

\section{Participants}

The participants consisted of 122 patients who were (a) consecutively referred to a UK specialist mood disorders research treatment center between January 2004 and 
March 2007, and (b) assessed for their suitability for either group mindfulness-based cognitive behavior therapy (MBCT) for relapse prevention or for individual cognitive behavior therapy (CBT) to treat current and residual depression. The patients met diagnostic criteria for current major depression $(n=37)$, past major depression $(n=100)$, dysthymia $(n=7)$ or bipolar disorder $(n=6)$ on the Structured Clinical Interview for DSM-IV (SCID, Spitzer et al. 1996). The patients with a diagnosis of bipolar disorder were excluded from all subsequent analyses and reports in order to maintain a homogeneous unipolar depressed sample. For the remaining 116 patients (41 male, 75 female), the mean score on the Beck Depression Inventory-II (BDI-II: Beck et al. 1996) was 29.42 (SD $=12.39$, range $0-60, n=113)$, mean age was $43.40(\mathrm{SD}=12.28$, range $19-73, n=115)$ and mean score on the Response Styles Questionnaire was 59.03 (SD $=12.37$, range $25-85, n=99)$. The mean number of previous episodes of major depression was 5.99 ( $\mathrm{SD}=4.3$, counting too many to count as a maximum of 9) and the mean age for the first onset of depression was 27.1 ( $\mathrm{SD}=13.2$ ). Within this sample, comorbid Axis I diagnoses included social anxiety $(n=33)$, GAD $(n=39)$, panic disorder with/without agoraphobia $(n=27)$, OCD $(n=14)$, PTSD $(n=10)$, past history of alcohol or substance misuse $(n=22)$, current alcohol or substance misuse $(n=5)$, and bulimia $(n=5)$. Co-morbid Axis II diagnoses included avoidant personality disorder $(n=25)$, obsessive-compulsive personality disorder $(n=12)$, depressive personality disorder $(n=12)$, paranoid personality disorder $(n=8)$, dependent personality disorder $(n=5)$, borderline personality disorder $(n=3)$, and passive-aggressive personality disorder $(n=2)$.

Materials

\section{Aide to History Taking Questionnaire}

All participants completed a written aide to history taking questionnaire prior to (and as a prerequisite for) an interview assessment. The aide to history questionnaire asked participants to report personal details (age, gender, occupation, marital status, religion), their family background, relationship with their parents, childhood and adolescence, work history, current relationship, psychiatric history, medical history, and alcohol and drug use history. To assess experiences of abuse, the questionnaire included a series of questions asking "Have you ever experienced X as a child?" and "Have you ever experienced X as an adult?", where X was, respectively, "physical abuse" "sexual abuse", "rape", "emotional or verbal abuse". The analysis was based on the comparison of Yes and No responses.

\section{Beck Depression Inventory-II (BDI-II; Beck et al. 1996)}

The BDI assesses levels of depressive symptoms with 21 items that are rated on a scale from 0 to 3 , with higher scores reflecting more depressive symptoms (range 0-63). Cronbach's alpha in our sample was .91. 
Response Styles Questionnaire-Ruminative Responses Scale (RSQ; Nolen-Hoeksema and Morrow 1991)

The RSQ measures the extent to which individuals generally respond to feeling sad, down or depressed by focusing on self, symptoms and on the causes and consequences of their mood, with 22-items rated on a 4-point frequency scale, from 1 (almost never) to 4 (almost always). Cronbach's alpha in our sample was .91. We also calculated scores on the separate brooding $(\alpha=.78)$ and reflection $(\alpha=.76)$ subscales identified by Treynor et al. (2003).

Beck Anxiety Inventory (BAI; Beck et al. 1988)

The BAI is a 21 item self-report scale assessing symptoms of anxiety for the past week (e.g., nervous, shaky, difficulty breathing, faint), with each item scored on a 0 (None, It did not bother me at all) to 3 (Severely, I could barely stand it) scale (range 0-63).

Penn State Worry Questionnaire (PSWQ; Meyer et al. 1990)

The PSWQ assesses trait pathological worry with 16 items (e.g., "I am always worrying about something"), rated from 1 (not at all typical of me) to 5 (very typical of me).

\section{SCID-II Personality Questionnaire (First et al. 1994)}

The SCID-II questionnaire is a 119 item self-report instrument designed to screen for Axis-II personality disorders. Participants are asked to answer each question in terms of "the kind of person you generally are-that is, how you have usually felt or behaved over the past several years." The questions ask about each of the diagnostic criteria for each personality disorder, e.g., for borderline personality disorder, questions include, "have you often become frantic when you thought that someone you really cared about was going to leave you?", "does your sense of who you are often change dramatically?", "have you often done things impulsively?". As well as a screening instrument for fuller SCID-II interviewing, questionnaire scores were used as a dimensional index of dysfunctional interpersonal, behavioral, and emotional traits associated with each disorder, scoring each question 1 (Yes) or 0 $(\mathrm{No})$.

The Structured Clinical Interview for DSM-IV (SCID; Spitzer et al. 1996)

The SCID for Axis I and II diagnoses was administered by an experienced clinician or a trained research worker for both current and past diagnoses. We have found high levels of reliability in diagnosis between independent raters trained in our clinic ( $\kappa$ of at least 0.8 ). 


\section{Procedure}

When referred to the research clinic, all participants were sent a consent form, the Aide to History Questionnaire and the self-report questionnaires. An assessment interview, consisting of further exploration of issues raised in the Aide to History questionnaire and the full SCID, was scheduled and conducted once these forms were returned.

\section{Results}

An alpha level of .05 was used for all statistical tests, although where appropriate to control for multiple comparisons, this value was adjusted using the Bonferroni procedure. All analyses were two-tailed. Different sample sizes for analyses reflect missing data on each questionnaire.

How is Rumination and its Subtypes Related to Different Symptoms (Depression and Anxiety)?

As a preliminary examination of hypotheses (a) and (e), the correlations between RSQ total score, brooding, reflection, BDI score, BAI score, and PSWQ score were calculated. These correlations are reported in Table 1. As predicted, rumination is significantly positively correlated with depression, anxiety and worry, replicating and extending previous findings in non-clinical (Fresco et al. 2002) or small clinical samples (Segerstrom et al. 2000). Given the significant positive correlation between depression and anxiety, the correlation between rumination and anxiety score was re-examined once depression score was partialled out, and vice versa. There was still a significant correlation between rumination and anxiety, even when depression was partialled out, $r=.33, p<.001, n=91$, and between rumination and depression once anxiety was partialled out, $r=.205, p=.041, n=91$. Thus,

Table 1 Correlation matrix for main variables

\begin{tabular}{lllllll}
\hline & Rumination & Brooding & Reflection & BDI & BAI & PSWQ \\
\hline Rumination & 1 & $.85^{* * *}$ & $.73 * * *$ & $.40^{* * *}$ & $.44 * * *$ & $.46^{* * * *}$ \\
Brooding & $.85 * * *$ & 1 & $.51 * * *$ & $.34 * * *$ & $.43 * * *$ & $.45^{* * * *}$ \\
Reflection & $.73 * * *$ & $.51 * * *$ & 1 & .16 & $.29 * *$ & $.29 * *$ \\
BDI & $.40^{* * *}$ & $.34 * * *$ & .16 & 1 & $.53 * * *$ & $.41^{* * *}$ \\
BAI & $.44 * * *$ & $.43 * * *$ & $.29 * *$ & $.53 * * *$ & 1 & $.30^{* * *}$ \\
PSWQ & $.46^{* * *}$ & $.45^{* * *}$ & $.29 * *$ & $.41 * * *$ & $.30^{* *}$ & 1 \\
\hline
\end{tabular}

Note: Rumination is the total score on the rumination subscale of the Response Style Questionnaire, $B D I$ Beck Depression Inventory, BAI Beck Anxiety Inventory, PSWQ Penn State Worry Questionnaire. Sample sizes range from $n=84$ to $n=110$ depending on missing data

$* p<.05 ; * * p<.01 ; * * * p<.001$ 
rumination appears to have a distinct and independent relationship with anxiety and depression.

Moreover, whereas brooding was significantly positively correlated with both depression and anxiety, reflection was only significantly correlated with anxiety. This finding replicates and extends to a clinically depressed sample Treynor et al.'s (2003) finding that the correlations between brooding and BDI are higher than those between reflection and BDI. Given the significant positive correlation between brooding and reflection, we further examined the correlation between each subtype of rumination and the symptom measures, once the other subtype of rumination was partialled out. Once brooding was partialled out, reflection was not significantly correlated with BAI, $r=.022, p=.83$; BDI, $r=-.057, p=.59$, (both $n=91$ ); or PSWQ, $r=.055, p=.64, n=81$, whereas with reflection partialled out, brooding was significantly correlated with BAI, $r=.39, p<.001$; BDI, $r=.33$, $p<.001$, and PSWQ, $r=.37, p<.001$. Thus, as predicted, brooding is more closely associated with increased levels of psychopathology than reflection.

Is Rumination a Transdiagnostic Process Associated with Co-Morbid Axis I Diagnoses?

To further test the hypothesis that rumination may be a transdiagnostic process with implications for disorders co-mordid to depression, separate logistic regression analyses were conducted for the presence or absence of each Axis I diagnosis with more than ten cases within this sample, with rumination as the independent variable, controlling for depression. The total rumination score was not significantly related to the presence or absence of any co-morbid diagnosis after controlling for current depression (all Wald $<2.7)$, except for a trend towards OCD status $(\beta=.073$, Wald $=3.36, p=.067)$. After controlling for current depression, brooding was significantly related to GAD status $(\beta=.188$, Wald $=6.79, p=.009)$ and OCD status $(\beta=.268$, Wald $=4.34, p=.04)$ but not to any of the other diagnoses (all Wald $<1.2, p>.28$ ). Brooding remains significantly related to GAD status, even after controlling for PSWQ and BDI $(\beta=.19$, Wald $=5.77, p=.012)$. Reflection was not significantly related to any diagnosis (all Wald $<2.5, p>.1$ ). Thus, increased brooding was associated with increased likelihood of having a co-morbid diagnosis of GAD and OCD, consistent with the transdiagnostic hypothesis.

\section{Is Rumination a Transdiagnostic Process Associated with Particular Personality Dysfunction?}

To examine the relationship between rumination and Axis II pathology, separate logistic regression analyses were conducted on the presence vs. the absence of any Axis II diagnosis, controlling for current depression, with each subtype of rumination as the independent variable. The diagnosis of personality disorder was not related to rumination $(\beta=.006$, Wald $=.12, p=.73)$, brooding $(\beta=.025$, Wald $=.17, p=.68)$ or reflection $(\beta=.015$, Wald $=.062, p=.80)$.

To test the hypothesis that rumination may be a transdiagnostic process with implications for Axis II conditions co-morbid to depression, separate logistic 
regression analyses were conducted for the presence or absence of each personality disorder diagnosis that had more than ten cases within our sample (avoidant, obsessive-compulsive, depressive), controlling for level of depression, with each subtype of rumination as the independent variable. Rumination, brooding and reflection were not associated with any of these specific personality disorders (all Wald $<1.8, p>.15$ ).

To further test whether rumination would be related to specific personality dimensions, the correlations between each dimension score and each subtype of rumination were calculated, after partialling out depression. Rumination was only significantly correlated with the borderline personality trait dimensions $(r=.37$, $p=.002, n=64)$. Brooding was significantly correlated with the borderline $(r=.30, p=.013, n=64)$, and obsessive-compulsive $(r=.23, p=.04, n=80)$ dimensions. Reflection was significantly correlated with the borderline $(r=.37$, $p=.002, n=66)$, passive-aggressive $(r=.27, p=.012, n=81)$, and obsessivecompulsive dimensions $(r=.23, p=.031, n=85)$. However, after using Bonferroni corrections to adjust the significance level for multiple correlations ( $\alpha$ adjusted to .004) only the correlations with the borderline dimensions remains significant. The questions assessing borderline personality reflect different elements within the diagnosis: instability of interpersonal relationships and self-image (e.g. "have you often become frantic when you thought that someone you really cared about was going to leave you?", "does your sense of who you are often change dramatically?") and marked impulsivity and extreme emotional responses (e.g., "have you often done things impulsively?", "do you often have a temper outbursts or get so angry that you lose control?"). As a post-hoc analysis, separate dimension scores for instability of relationships/self-image and impulsivity/extreme emotional response were calculated. After partialling out depression, instability of relationships/self-image was significantly correlated with rumination $(r=.45, p=.001$, $n=64)$, brooding $(r=.39, p=.001, n=64)$, reflection $(r=.45, p=.001$, $n=64)$, whereas impulsivity/extreme emotional response was not correlated with any of the subtypes of rumination (all $r<.14, p>.27$ ).

Is Rumination a Vulnerability Trait or State-Dependent?

To test the hypothesis that rumination has trait-like qualities rather than simply being state dependent, separate Analyses of Variance (ANOVAs) by Group (currently depressed CD vs. formerly depressed FD) were calculated for each subtype of rumination (total RSQ, brooding, reflection) as the dependent variable. There was no significant difference between the groups on rumination score, $F(1,92)=.03, p=.87(\mathrm{CD}: M=59.19, \mathrm{SD}=11.32, n=31 ; \mathrm{FD}: M=58.75$, $\mathrm{SD}=12.63, n=63)$, brooding, $F(1,92)=.18, \quad p=.68 \quad(\mathrm{CD}: M=13.22$, $\mathrm{SD}=3.41 ; \mathrm{FD}: M=13.57, \mathrm{SD}=3.90)$ or reflection score, $F(1,92)=.014$, $p=.91$ (CD: $M=11.77, \mathrm{SD}=3.93$; FD: $M=11.68, \mathrm{SD}=3.32$ ). Critically, there was a significant difference between the groups in level of depressive symptoms on the BDI score, $F(1,87)=14.33, p<.001$, reflecting higher BDI scores in the $\mathrm{CD}$ group $(M=36.40, \mathrm{SD}=8.29, n=30)$ than in the FD group $(M=27.24, \mathrm{SD}=11.85, n=59)$. This pattern of results suggests that, as 
predicted, the level of rumination is not simply dependent on mood state and clinical status, but rather may have more trait-like qualities, such that it remains elevated even when participants are no longer in an episode of major depression.

\section{Is Rumination Elevated in Women Compared to Men?}

To test the hypothesis that rumination is more prevalent in women than men, separate ANOVAs were calculated by Gender for each subtype of rumination as the dependent variable. Contrary to previous findings, there was no difference by gender for rumination score, $F(1,97)=1.19, p=.28$ (men, $M=57.31, \mathrm{SD}=$ $11.39, n=38$; women $M=60.10, \mathrm{SD}=12.92, n=61)$, brooding, $F(1,97)=$ $1.19, p=.28$ (men, $M=12.95, \mathrm{SD}=3.80$; women, $M=13.79, \mathrm{SD}=3.67$ ), and reflection, $F(1,97)=.14, p=.71$ (men, $M=11.58, \mathrm{SD}=3.31$; women, $M=11.85, \mathrm{SD}=3.69$ ).

\section{Is Rumination Related to Reported History of Abuse?}

To examine the relationship between rumination and reports of abuse, logistic regressions were conducted on the self-report of the presence (Yes) vs. the absence $(\mathrm{No})$ of each type of abuse, controlling for depression, with each subtype of rumination as separate independent variables. Because the current indices were categorical (yes or no to report of abuse) rather than measures of frequency as in the Conway et al. (2004) study, the report of child sexual abuse and report of adult rape were first combined into a report of any sexual abuse to increase the sensitivity of the measure. As predicted, after controlling for depression, report of any sexual abuse was significantly related to rumination $(\beta=.060$, Wald $=4.91, p=.027)$ and nearly significantly related to brooding $(\beta=.151$, Wald $=3.42, p=.064)$ but not related to reflection $(\beta=.097$, Wald $=1.85, p=.17)$. When investigated separately, after controlling for depression, report of adult rape was significantly related to rumination $(\beta=.078$, Wald $=5.12, p=.024)$ and report of childhood sexual abuse was nearly significantly related to rumination $(\beta=.053$, Wald $=3.29$, $p=.07)$. However, brooding was not significantly correlated with report of childhood sexual abuse $(\beta=.16$, Wald $=2.60, p=.11)$ or adult rape $(\beta=.110$, Wald $=1.39, p=.24)$. No other regressions were significant $($ Wald $<2)$, although there was a trend for report of adult physical abuse to be related to rumination $(\beta=.043, \quad$ Wald $=2.78, \quad p=.095)$ and brooding $(\beta=.15, \quad$ Wald $=3.09$, $p=.079)$ and for report of childhood physical abuse to be related to reflection $(\beta=.13$, Wald $=3.09, p=.079)$.

\section{Discussion}

The current study tested whether a number of rumination-related findings in nonclinical samples could be replicated and extended to a clinical sample of depressed patients, with a particular focus on testing whether rumination was related to dimensions of psychopathology and diagnoses co-morbid with depression, 
consistent with the transdiagnostic hypothesis. The majority of previous findings were replicated, with the exception that the predicted gender difference in rumination was not observed.

First, the current findings provide further evidence consistent with the hypothesis that rumination may be a transdiagnostic process involved in psychological disorders other than depression. Rumination was independently associated with both anxiety and depression. Further, brooding was significantly associated with the diagnoses of OCD and GAD, even after controlling for depression. Unfortunately, there were insufficient cases of PTSD, bulimia and substance abuse in the current sample to test whether relationships between rumination and these disorders could be replicated. Contrary to prediction, despite a high frequency of co-morbidity in this sample, social anxiety was not associated with rumination. This was unexpected given the growing literature reporting that ruminative thinking in the form of postmortems analyzing past social interactions is characteristic of social anxiety (e.g., Abbott and Rapee 2004; Mellings and Alden 2000).

Also consistent with rumination being related to dysfunction other than depression, the current study provides the first replication of Smith et al. (2006) findings that rumination was related to the dysfunctional borderline dimension (and not related to personality disorder diagnosis). Since the Smith et al. (2006) study used undergraduates varying in cognitive risk for depression but with no current Axis I disorders, the current findings establish that this relationship between rumination and borderline traits still holds true in a clinically depressed sample. The current study suggested that there was a trend for rumination to be related to obsessive-compulsive personality dysfunction but this was not significant after adjusting for multiple correlations. Interestingly, Smith et al. (2006) found that obsessive-compulsive dimension scores no longer correlated with rumination once "cognitive preoccupation" items conceptually similar to rumination were removed. Thus both studies suggest that only the borderline dimension is reliably correlated with rumination.

Interestingly, rumination was associated with a specific subset of borderline traits: with instability and inconsistency within relationships, identity, self-image, goals, career, and plans, but not with impulsiveness, lack of control of anger or selfharm. Because the results are correlational, the causal relationship between rumination and this instability of identity and self-image is not known. Nonetheless, I speculate that the causal relationship may be bidirectional. Because rumination can be triggered by unresolved personal concerns and unattained goals (Martin and Tesser 1989, 1996), and because inconsistency in goals and values is likely to interfere with progress towards goals and produce contradictory and conflicting goals, instability of self-identity may cause rumination. Moreover, the recurrent dwelling on self and problems within rumination can lead to repeated questioning and doubting (e.g. "Why can't I handle things better?"), which could cause instability of self-image. Experimental manipulations of rumination and goals could usefully disentangle the causal relationship between rumination and instability of identity.

Second, the present findings are consistent with the previous finding that previously dysphoric students have similar levels of rumination to currently 
dysphoric students (Roberts et al. 1998). Importantly, whereas Roberts et al. (1998) used an undergraduate sample and defined the presence or absence of dysphoria with reference to the DSM-IV symptom criteria for major depression but not with reference to the 2-week duration criterion, the current study used a clinically referred patient sample who met full diagnostic criteria for current or past major depression. These results therefore indicate that clinical status does not account for level of rumination in a real-world patient sample, further confirming that rumination is not simply state-dependent, but, rather, might have trait-like characteristics.

Third, the present findings failed to replicate the repeated finding within undergraduate and community samples that rumination is elevated in women relative to men. This result suggests that this pattern does not generalize to a sample of patients with recurrent histories of depression seeking specialist psychological help. It may be that in those individuals who have experienced frequent depression, rates of rumination are elevated regardless of gender, either because elevated rumination is a predisposition that confers vulnerability to subsequent depression or because repeated experience of depression exacerbates rumination.

Fourth, the current findings provide further evidence that that brooding is more associated with psychopathology than reflection. Brooding was significantly correlated with level of depression whereas reflection was not correlated with depression. After controlling for each other, brooding was still significantly correlated with anxiety, depression and worry, whereas reflection was not. Brooding was associated with report of sexual abuse but reflection was not. Together, all of these findings provide further evidence consistent with Treynor et al.'s (2003) proposal that brooding is a more maladaptive subtype of rumination than reflection, and extend this finding to a depressed sample.

Fifth, despite only indexing Yes or No reports about abuse, rather than measuring frequency of unwanted sexual activity, the current study replicated Conway et al's (2004) finding that reports of sexual abuse are correlated with increased rumination. More specifically, closely paralleling Conway et al.'s (2004) findings, rumination was related to report of adult rape and to report of child sexual abuse. Although these reports of abuse cannot establish causality, they are consistent with the hypothesis that sexual abuse/victimization may contribute to the development of rumination. Since abuse often involves painful, hard-to-understand experiences, powerlessness, and prohibitions from talking about what happened to others, it is a plausible hypothesis that it would produce turning inwards, passivity, and repeated attempts to analyze and understand what happened, and, thereby, exacerbate rumination.

\section{Assessment and Treatment of Rumination}

These findings have a number of implications for the identification, assessment and treatment of rumination within depression. Foremost, it appears that rumination can be equally elevated in both men and women with histories of recurrent depression. This result suggests that a detailed assessment of rumination should be undertaken in depressed patients regardless of gender. Second, these results suggest that rumination is likely to be more elevated in depressed patients with more severe 
depression and anxiety, reports of sexual abuse, co-morbid GAD and/or OCD, unstable relationships, and confusion or inconsistency concerning goals, priorities and values. Thus, where these elements form part of the clinical presentation, it may be useful to also assess for rumination; likewise for patients who present with high levels of rumination, it may be useful to assess these associated elements, since knowledge of these different issues may inform treatment.

These findings suggest a number of potential treatment options for depressive rumination. First, the association between report of abuse and rumination suggests the hypothesis that for a patient who reports past sexual abuse or rape, intrusive memories and thoughts about these traumas may underpin and contribute to the rumination and associated depression. This association therefore suggests that interventions focused on exposure and cognitive restructuring to resolve the traumatic response to these events (e.g., Resick and Scnicke 1992) could be a beneficial intervention. Second, the relation between rumination and borderline personality dimensions suggests that CBT interventions specifically developed to treat borderline personality disorder (Beck and Freeman 1990; Linehan 2003) could be usefully adapted to help in the treatment of depressive rumination, particularly by reducing instability of interpersonal relationships and identity. Consistent with this, mindfulness meditation, an integral part of dialectical behavior therapy for borderline personality disorder, has recently been demonstrated to reduce rumination (Ramel et al. 2004). The relationship between borderline personality dimensions and rumination also suggests the importance of developing and maintaining a good collaborative therapeutic relationship with ruminators. The pattern of unstable and intense relationships observed in the borderline dimension can also play out in the therapeutic relationship, so that care may need to be taken to be consistent and clear in communications to avoid loss of trust. Third, the differential findings between brooding and reflection further emphasize that there may be distinct subtypes of rumination with distinct functional consequences. One treatment implication of this finding is that it may be beneficial to shift patients from more maladaptive to less maladaptive forms of rumination, rather than attempting to halt rumination altogether.

A recently developed variant of CBT designed to explicitly target rumination (Rumination-focused CBT) includes both a focus on shifting patients to more adaptive forms of rumination and a focus on reducing instability and inconsistency in identity and self-image (Watkins et al. 2007). RFCBT uses functional analysis of the differences between bouts of helpful vs. unhelpful rumination, as well as experiential/imagery exercises and behavioral experiments to facilitate a shift into a more helpful form of rumination. Patients use directed imagery to vividly recreate previous states when a more helpful thinking style was active, such as memories of being completely absorbed in an activity (e.g., "flow" or "peak" experiences). RFCBT also explicitly uses guided discovery with patients to identify their own personally meaningful values and to separate their own values from values that have been imposed externally or internalized from authority figures. Patients are then encouraged to act in ways that are consistent with and that serve their own values, to strengthen their own sense of identity. Consistent with the potential benefit of these approaches, a multiple-baseline case series of 14 patients with residual depression 
has provided preliminary evidence that this treatment can be effective at reducing depressive symptoms and rumination (Watkins et al. 2007).

Despite its utility, several limitations of the present study must be acknowledged. The sample consisted of patients with chronic and recurrent depression referred for specialist treatment, and, therefore the findings may not be generalizable to other populations. In particular, depressive symptoms were still high even in the formerly depressed group. Nonetheless, this clinical sample provides a useful counterpoint to studies that have focused mainly on undergraduates. Despite being a clinical sample, several Axis I and Axis II disorders were relatively infrequent, preventing an assessment of the relationship between rumination and these diagnoses. Most notably, there were few diagnoses of borderline personality disorder: rumination may be found to be related to borderline personality disorder when this diagnosis is more frequent. Finally, because the results are correlational and cross-sectional, no causal conclusions can be drawn, emphasizing the need for longitudinal studies to determine whether rumination can prospectively predict co-morbidity and whether report of abuse predicts rumination.

In sum, these results suggest that key findings in the rumination literature can be replicated in a clinical sample. Most importantly, these results provide further evidence consistent with the hypothesis that rumination is a transdiagnostic process: rumination is independently related to both depression and anxiety; brooding is related to co-morbid $\mathrm{OCD}$ and GAD in depressed patients, and rumination is associated with the borderline personality dimension.

Acknowledgement This research was supported by a project grant (08099/Z/06/Z) from the Wellcome Trust, UK, awarded to Dr Edward R. Watkins.

Open Access This article is distributed under the terms of the Creative Commons Attribution Noncommercial License which permits any noncommercial use, distribution, and reproduction in any medium, provided the original author(s) and source are credited.

\section{References}

Abbott, M. J., \& Rapee, R. M. (2004). Post-event rumination and negative self-appraisal in social phobia before and after treatment. Journal of Abnormal Psychology, 113, 136-144.

Abela, J. R. Z., Payne, A. V. L., \& Moussaly, N. (2003). Cognitive vulnerability to depression in individuals with borderline personality disorder. Journal of Personality Disorders, 17, 319-329.

American Psychiatric Association. (1994). Diagnostic and statistical manual of mental disorders (4th ed.). Washington DC: Author.

Beck, A. T., Brown, G., Epstein, N., \& Steer, R. A. (1988). An inventory for measuring clinical anxietypsychometric properties. Journal of Consulting and Clinical Psychology, 56, 893-897.

Beck, A. T., \& Freeman, A. (1990). Cognitive therapy of personality disorders. New York: Guilford Press.

Beck, A. T., Steer, R. A., \& Brown, G. K. (1996). The Beck Depression Inventory (2nd ed.). San Antonio, TX: The Psychological Corporation.

Butler, L. D., \& Nolen-Hoeksema, S. (1994). Gender differences in responses to depressed mood in a college sample. Sex Roles, 30, 331-346.

Conway, M., Mendelson, M., Giannopoulos, C., Csank, P. A. R., \& Holm, S. L. (2004). Childhood and adult sexual abuse, rumination on sadness, and dysphoria. Child Abuse and Neglect, 28, 393-410.

Ehlers, A., Mayou, R. A., \& Bryant, B. (1998). Psychological predictors of chronic posttraumatic stress disorder after motor vehicle accidents. Journal of Abnormal Psychology, 107, 508-519. 
First, M. B., Spitzer, R. L., Gibbon, M., Williams, J. B. W., \& Benjamin, L. (1994). Structured clinical interview for DSM-IV axis II personality disorders (SCID-II), (Version 2.0). New York: New York State Psychiatric Institute.

Fresco, D. M., Frankel, A. N., Mennin, D. S., Turk, C. L., \& Heimberg, R. G. (2002). Distinct and overlapping features of rumination and worry: The relationship of cognitive production to negative affective states. Cognitive Therapy and Research, 26, 179-188.

Harvey, A. G., Watkins, E., Mansell, W., \& Shafran, R. (2004). Cognitive behavioural processes across psychological disorders. Oxford: Oxford University Press.

Hong, R. Y. (2007). Worry and rumination: Differential associations with anxious and depressive symptoms and coping behavior. Behaviour Research and Therapy, 45, 277-290.

Hoyer, J., Becker, E. S., \& Margraf, J. (2002). Generalized anxiety disorder and clinical worry episodes in young women. Psychological Medicine, 32, 1227-1237.

Kasch, K. L., Klein, D. N., \& Lara, M. E. (2001). A construct validation study of the response styles questionnaire rumination scale in participants with a recent-onset major depressive episode. Psychological Assessment, 13, 375-383.

Linehan, M. M. (2003). Cognitive-behavioural treatment of borderline personality disorder. New York: Guilford Press.

Lyubomirsky, S., \& Nolen-Hoeksema, S. (1995). Effects of self-focused rumination on negative thinking and interpersonal problem-solving. Journal of Personality and Social Psychology, 69, 176-190.

Martin, L. L., \& Tesser, A. (1989). Toward a motivational and structural theory of ruminative thought. In J. S. Uleman \& J. A. Bargh (Eds.), Unintended thought (pp. 306-326). New York: Guilford Press.

Martin, L. L., \& Tesser, A. (1996). Some ruminative thoughts. In R. S. Wyer (Ed.), Ruminative thoughts. Advances in social cognition (Vol. 9, pp. 1-47). Hillsdale, NJ: Lawrence Erlbaum Associates.

Mayou, R. A., Ehlers, A., \& Bryant, B. (2002). Posttraumatic stress disorder after motor vehicle accidents: 3-year follow-up of a prospective longitudinal study. Behaviour Research and Therapy, 40, 665-675.

Mellings, T. M. B., \& Alden, L. E. (2000). Cognitive processes in social anxiety: The effects of selffocus, rumination and anticipatory processing. Behaviour Research and Therapy, 38, 243-257.

Meyer, T. J., Miller, M. L., Metzger, R. L., \& Borkovec, T. D. (1990). Development and validation of the Penn State Worry Questionnaire. Behaviour Research and Therapy, 28, 487-495.

Nolen-Hoeksema, S. (1991). Responses to depression and their effects on the duration of depressive episodes. Journal of Abnormal Psychology, 100, 569-582.

Nolen-Hoeksema, S. (2000). The role of rumination in depressive disorders and mixed anxiety/depressive symptoms. Journal of Abnormal Psychology, 109, 504-511.

Nolen-Hoeksema, S., \& Jackson, B. (2001). Mediators of the gender difference in rumination. Psychology of Women Quarterly, 25, 37-47.

Nolen-Hoeksema, S., Larson, J., \& Grayson, C. (1999). Explaining the gender difference in depressive symptoms. Journal of Personality and Social Psychology, 77, 1061-1072.

Nolen-Hoeksema, S., \& Morrow, J. (1991). A prospective-study of depression and posttraumatic stress symptoms after a natural disaster-the 1989 Loma-Prieta Earthquake. Journal of Personality and Social Psychology, 61, 115-121.

Nolen-Hoeksema, S., \& Morrow, J. (1993). Effects of rumination and distraction on naturally-occurring depressed mood. Cognition \& Emotion, 7, 561-570.

Nolen-Hoeksema, S., Stice, E., Wade, E., \& Bohon, C. (2007). Reciprocal relations between rumination and bulimic, substance abuse, and depressive symptoms in female adolescents. Journal of Abnormal Psychology, 116, 198-207.

Ramel, W., Goldin, P. R., Carmona, P. E., \& McQuaid, J. R. (2004). The effects of mindfulness meditation on cognitive processes and affect in patients with past depression. Cognitive Therapy and Research, 28, 433-455.

Resick, P. A., \& Scnicke, M. K. (1992). Cognitive processing therapy for sexual assault victims. Journal of Consulting and Clinical Psychology, 60, 748-756.

Rimes, K. A., \& Watkins, E. (2005). The effects of self-focused rumination on global negative selfjudgements in depression. Behaviour Research and Therapy, 43, 1673-1681.

Roberts, J. E., Gilboa, E., \& Gotlib, I. H. (1998). Ruminative response style and vulnerability to episodes of dysphoria: Gender, neuroticism, and episode duration. Cognitive Therapy and Research, 22, 401-423.

Sarin, S., Abela, J. R. Z., \& Auerbach, R. P. (2005). The response styles theory of depression: A test of specificity and causal mediation. Cognition \& Emotion, 19, 751-761. 
Segerstrom, S. C., Tsao, J. C. I., Alden, L. E., \& Craske, M. G. (2000). Worry and rumination: Repetitive thought as a concomitant and predictor of negative mood. Cognitive Therapy and Research, 24, 671-688.

Smith, J. M., Grandin, L. D., Alloy, L. B., \& Abramson, L. Y. (2006). Cognitive vulnerability to depression and axis II personality dysfunction. Cognitive Therapy and Research, 30, 609-621.

Spasojevic, J., \& Alloy, L. B. (2001). Rumination as a common mechanism relating depressive risk factors to depression. Emotion, 1, 25-37.

Spitzer, R. L., Williams, J. B. W., Gibbon, M., \& First, M. B. (1996). Structured clinical interview for DSM-IV (SCID). Washington DC: American Psychiatric Association.

Treynor, W., Gonzalez, R., \& Nolen-Hoeksema, S. (2003). Rumination reconsidered: A psychometric analysis. Cognitive Therapy and Research, 27, 247-259.

Vanoppen, P., Hoekstra, R. J., \& Emmelkamp, P. M. G. (1995). The structure of obsessive-compulsive symptoms. Behaviour Research and Therapy, 33, 15-23.

Watkins, E., \& Moulds, M. (2005). Distinct modes of ruminative self-focus: Impact of abstract versus concrete rumination on problem solving in depression. Emotion, 5, 319-328.

Watkins, E. R., Scott, J., Wingrove, J., Rimes, K. A., Bathurst, N., Steiner, H., et al. (2007). Ruminationfocused cognitive behaviour therapy for residual depression: A case series. Behaviour Research and Therapy, 45, 2144-2154.

Watkins, E., \& Teasdale, J. D. (2001). Rumination and overgeneral memory in depression: Effects of selffocus and analytic thinking. Journal of Abnormal Psychology, 110, 353-357.

Watkins, E., \& Teasdale, J. D. (2004). Adaptive and maladaptive self-focus in depression. Journal of Affective Disorders, 82, 1-8. 